\title{
Evaluation of a Thermoelectric Generation system based on Differential-Power Processing architecture under non-uniform temperature conditions
}

\author{
Jesús Sergio Artal-Sevil, Carlos Bernal-Ruiz \\ Department of Electrical Engineering \\ University of Zaragoza \\ Zaragoza, Spain \\ jsartal@unizar.es; cbernal@unizar.es
}

\author{
Jesús Beyza, Victor M. Bravo \\ Department of Electromechanical \\ Costa Grande Institute of Technology \\ Zihuatanejo, Mexico \\ jbeyza@unizar.es; viictoor2020@hotmail.com
}

\begin{abstract}
This paper presents a Differential Power Processing (DPP) architecture applied to series-connected thermoelectric generators (TEG). Currently, thermoelectric technology is being considered as a promising power generation technology that can be used to recover waste heat energy. Thus, a thermoelectric generation system is studied under non-uniform temperature conditions in multiple TEG devices. The main objective is to allow each thermoelectric sub-module to reach its maximum power point more quickly. The purpose has been to improve the maximum power point tracking (MPPT) in each sub-module, thus it is possible to increase the efficiency with respect to the traditional method based on a global MPPT. Differential Power converters have been implemented in each TEG sub-module to provide an effective solution and mitigate the impact of the mismatch in the power obtained. The DPP architecture consists of a small micro-converter, at the submodular level, applied to the thermoelectric cell. The control algorithm is oriented to polarize each TEG device at its optimal point, which allows us an active balancing among the different TEG sub-modules regardless of the operating temperature. Matlab-Simulink has been the software used to develop the TEG module-array.
\end{abstract}

Keywords-Thermoelectric Generator (TEG); TEG modelling; Differential-Power Processing (DPP); Maximum Power Point Tracking (MPPT); Waste heat recovery technologies; non-uniform temperature distribution; Energy Harvesting; DC-DC converter.

\section{INTRODUCTION}

In recent years, many scientists have focused on improving and optimizing power generators based on energy harvesting, in order to reduce global warming. Nowadays, a growing interest has appeared in society in recovering energy from some abandoned natural sources such as thermal, RF radiation, vibratory/mechanical energy, piezoelectric energy, etc., and its subsequent conversion into electrical energy for the purpose of supply electronic systems or low-power machinery.

Thermal energy is an available energy source that can be found in multiple applications, such as industrial processes, running vehicles, and even in domestic equipment, etc. Some devices that have attracted attention recently are thermoelectric generators (TEGs). These devices do not contain chemical products since they are manufactured with different types of substrates such as silicon, polymers, and ceramics, in this way they are friendly with the environment. Furthermore, these devices are simple as they do not contain moving parts or complex structures inside, being silent devices with a long operating lifetime. TEGs are composed of multiple thermocouples, electrically connected in series and thermally in parallel. Thus, thermoelectric generators have demonstrated their ability to directly convert thermal energy into electrical energy through the Seebeck effect. These devices are also bidirectional and can cool a surface by applying a voltage across its terminals (Peltier effect). Thus, Peltier coolers are used to convert electrical energy into thermal energy for cooling purposes.

Although the efficiency of these devices is not very high, they can be used in many fields due to their free maintenance and long service life. Thus, some authors have shown interest in its applications for energy harvesting. Jaziri et al. [1] present in-depth analysis of TEGs. In this way, they describe their operating principle, materials and technologies used, and even analyze different internal structures (conventional, cascaded and segmented). The authors mention different high-power applications such as industrial electronics, automotive engines, and aerospace. Similarly, Twaha et al. [2] develop a comprehensive review of thermoelectric technology. Its main contribution is associated with performance improvement of TEG devices through the study of new designs, geometries, the incorporation of different materials, as well as the use of new advanced mathematical models. Recent advances in materials, selection criteria and the main parameters related to TEG devices are presented. These techniques have facilitated the study and analysis of TEG modules. The recovery and utilization of waste heat can be an option to obtain a certain amount of energy and thus reduce energy consumption. In turn, this strategy allows us to improve the overall efficiency of the systems. However, the optimal performance of the different TEG modules depends on several factors such as material selection and operating strategy. Although thermoelectric devices contain many advantages due to their compact structure, their applications have not been widely developed due to their low efficiency. 
Thermoelectric technology is being seen as a promising power generation technology that can be used to recover waste heat energy. In this way, TEG technology is being introduced in automotive engines to extract waste energy at different points in the vehicle, such as the exhaust pipe, cooling system, clutch, gearbox, etc., [3]. Thus, Hebert and Schwab [4] present a techno-economic approach to the modeling of a thermoelectric generator for natural gas heavy-duty vehicles. This time the TEG devices are integrated into the exhaust system. This paper can be complemented with the study carried out by Yan et al. [5] on performance analysis in TEG devices placed on channels and tubes with different cross-sectional shapes. In all these cases the objective is to optimize the heat sources of internal combustion engines that use fossil fuels. Other studies have also been developed for the harvesting of thermoelectric energy from a railway track.

Thus Gao et al. [6] develop a thermoelectric prototype for capturing thermal energy on railway tracks. This technology enables the power supply to sensor-based systems in remote areas and off-grid modules. Similarly, Kütt et al. [7] analyze different domestic applications (boilers and ovens, biomass stoves, etc.) to study the capture of thermoelectric energy and the viability of different micro-production units. Meanwhile, Dewan et al. [8] propose TEG modules as alternative energy sources to supply remote sensor-based systems. Batteries are traditionally used as power sources in stand-alone systems and they have replacement cost as the main drawback. In this way, the authors provide thermoelectric harvesting techniques as a supply system in wireless sensor networks, depending on power requirements. Likewise, Wan et al. [9] perform the analysis and design of a novel energy harvesting system based on a reconfigurable TEG array for IoT applications.

Meanwhile, Hasani \& Rahbar [10] propose the application of a thermoelectric cooler as a possible option for the waste heat recovery in a system composed of a PEM fuel cell. Temperature is an important parameter in the optimal operation of the PEM fuel cell, as it affects both its efficiency and the maximum cell voltage. In this way, the introduction of multiple thermoelectric devices to support the temperature management system is presented. Moreover, these TEG devices can play an important role, for example in deep space probes as a reliable source of power [11]. The space industry has used TEG, in combination with thermal generators based on nuclear technology (radioisotope thermoelectric generators), since the beginning of space exploration. Medical and wearable devices, microgeneration for sensors, computer systems, and electricity generation in extreme environments are other possible applications of these thermoelectric devices.

On the other hand, the amount of energy obtained by a TEG depends on the difference in temperature and the applied electrical load. Thus, Bond et al. [12] or Kim et al. [13] present some maximum power point tracking (MPPT) methods applied to TEG devices. The proposed MPPT energy harvesting schemes are simple and have low power consumption. In this way, the maximum power can be extracted for each given temperature condition. Both papers use a DC-DC Boost converter to implement the MPPT algorithm. While in [14], the authors propose an MPPT scheme composed of a synchronous
Buck-Boost converter and where the step size can be modified. Similarly, Park et al. [15] propose a temperature sensor-based MPPT algorithm for energy harvesting. The purpose is to compensate for the low conversion efficiency. In turn, this technique can avoid some disadvantages associated with other more conventional methods such as device disconnection, current measurement or power disturbances. Likewise, in [16] the authors propose a new adaptive MPPT technique for a centralized thermoelectric generation system. At the same time, different configurations of TEG system (centralized, stringtype and modularized) are presented and analyzed.

In the present paper, a thermoelectric generation system is studied under non-uniform temperature conditions in TEG devices. In these conditions, with a series distribution of the TEG devices, multiple local maximum power points (LMMP) appear. A differential-power processing architecture [17] [18] is introduced in order to optimize waste energy harvesting.

This paper is organized as follows. Section 1 has shown a brief introduction and the state-of-the-art of the problem addressed. Section 2 gives a description of the system configuration. The basic principle of operation in TEG devices is also explained. Section 3 describes the differential-power converter and its control strategy. Maximum Power Point Tracking (MPPT) algorithm is presented. Section 4 shows the different results obtained from the simulation. Matlab-Simulink simulation software has been used. Finally, the conclusions and some brief considerations are described in Section 5 .

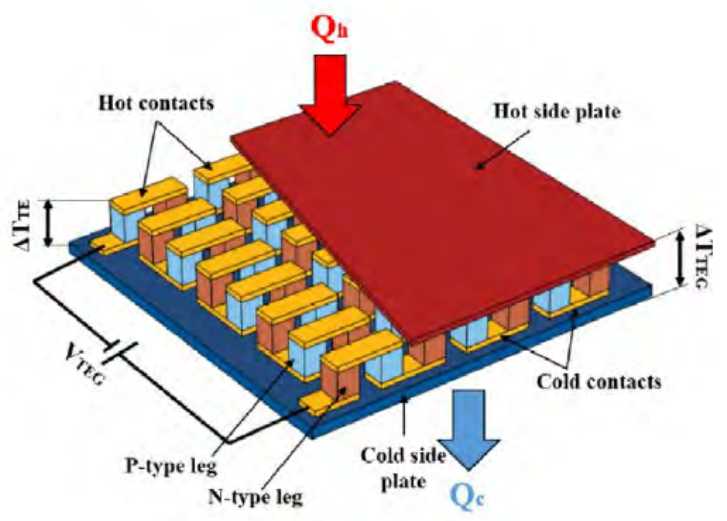

Fig. 1. Example of a thermoelectric device (TEG) and its different elements.

\section{Thermoelectric Generators (TEGs)}

Recently, among clean energy conversion technologies, thermoelectric devices have received a lot of attention because they are solid-state devices capable of converting wasted thermal energy in many applications into electrical energy. Thermoelectric modules are semiconductor devices that have the ability to generate a voltage when exposed to a temperature gradient (Seebeck effect), or on the contrary, to produce a temperature gradient when supplied by a voltage (Peltier effect). TEGs are generally composed of many thermocouples connected in series in order to increase the power output. Although thermoelectric devices have low efficiency and high cost, their viability as energy source has improved thanks to recent advances in research associated with material efficiency. 


\section{A. Basic principle}

Thermocouples are made of two different materials, which have an opposite Seebeck coefficient $\left(\alpha_{\mathrm{A}}, \alpha_{\mathrm{B}}\right)$, joined through their ends, (see Fig. 1). A good thermoelectric material has a Seebeck coefficient range between $\pm 350 \mu \mathrm{V} / \mathrm{K}[1]$, [7]. Thus, due to the Seebeck effect, the temperature gradient causes a voltage at its terminals $\left(v_{O C}\right)$ that can be expressed as:

$$
v_{O C}=N \alpha_{A B} \Delta T
$$

where $N$ is the number of thermocouples connected in series, $\alpha_{A B}$ is the Seebeck coefficient corresponding to the union of both semiconductor materials $A$ and $B\left(\alpha_{A B}=\alpha_{A}-\alpha_{B}\right)$, while $\Delta T$ is the temperature gradient between the surfaces that make up the TEG device $\left(\Delta \mathrm{T}=\mathrm{T}_{\mathrm{H}}-\mathrm{T}_{\mathrm{C}}\right)$; being the parameters $T_{H}$ and $T_{C}$, the temperature of the hot side and the cold side, respectively. Semiconductor materials generate a higher $v_{O C}$ compared to metals; hence they are used to manufacture TEG devices. In this way, a positive doped semiconductor (p-type) is connected through a metallic contactor to a negative doped semiconductor (n-type) to create a thermoelectric junction, considered a p-n pair, as shown in Fig. 1.

In steady state, a TEG can be modeled as a voltage source $v_{O C}$ in series with an internal electrical resistance $\left(R_{T E G}\right)$ as presented in Fig. 2. Note that both $v_{O C}$ and $R_{T E G}$ are highly dependent on the temperature difference. In turn, the total internal resistance of the module is proportional to the number of thermocouples connected in series. Thus, although a large number of thermocouples increase the voltage provided by the TEG device, their impact on internal resistance is adverse. In this assumption, the internal resistance $R_{T E G}$ of the TEG device can be expressed as:

$$
R_{T E G}=N\left(\frac{\rho_{A} L_{A}}{S_{A}}+\frac{\rho_{B} L_{B}}{S_{B}}+2 \frac{\rho_{C} L_{C}}{S_{C}}\right)=\sum_{i=1}^{n} R_{T J, i}
$$

where the different coefficients $\rho_{A}, \rho_{B}$ and $\rho_{C}$ are, respectively, the electrical resistivity of materials $\mathrm{A}, \mathrm{B}$ and of the metallic contact. Meanwhile, $L_{A}$ and $L_{B}$ are the lengths of the thermocouple arms crossed by the heat flux and $L_{C}$ is the length of the metallic contact. $S_{A}, S_{B}$, and $S_{C}$ are thermocouples $\mathrm{A}$ and $\mathrm{B}$ respectively, along with the cross-sectional areas of the metallic contacts; while $R_{T J, i}$ is the resistance of the ith thermocouple junction.

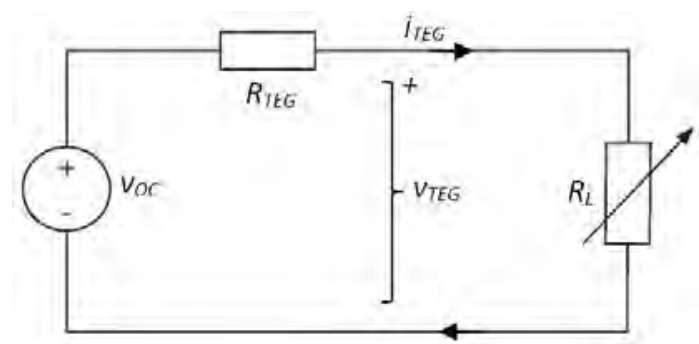

Fig. 2. Ideal electrical equivalent circuit of a TEG device.

\section{B. Electrical Model}

A TEG generator can be modelled as a voltage source, with an open-circuit voltage $\left(v_{O C}\right)$, and an internal series resistance
$\left(R_{T E G}\right)$. While the external load $\left(R_{L}\right)$ can be fixed or variable. Thevenin equivalent circuit of the TEG device is shown in Fig. 2. Through the equivalent circuit, it can be verified that the output current-voltage relationship $\left(v_{T E G}, i_{T E G}\right)$ is linear. When the TEG module operates with a load resistor $R_{L}$, the output voltage is given by:

$$
v_{T E G}=v_{O C} \frac{R_{L}}{R_{T E G}+R_{L}}=\frac{\left(N \alpha_{A B} \Delta T\right) R_{L}}{R_{T E G}+R_{L}}
$$

In this way, the output power delivered by the TEG generator $\left(P_{T E G}\right)$ will be given by:

$$
P_{T E G}=v_{O C}{ }^{2} \frac{R_{L}}{\left(R_{T E G}+R_{L}\right)^{2}}
$$

where $R_{L}$ and $R_{T E G}$ are the external load and internal resistance of the TEG device, respectively. Now the maximum power can be found by developing the derivative with respect to $R_{L}$.

$$
\frac{d P_{T E G}}{d R_{L}}=v_{O C}{ }^{2} \frac{\left(R_{T E G}-R_{L}\right)}{\left(R_{T E G}+R_{L}\right)^{3}}=0
$$

In this case, the maximum power output occurs for the condition $\mathrm{R}_{\mathrm{TEG}}=\mathrm{R}_{\mathrm{L}}$. The theoretical maximum power of the TEG module can now be expressed as:

$$
P_{T E G}^{\max }=\frac{v_{O C}{ }^{2}}{4 R_{T E G}}=\frac{\left(N \alpha_{A B} \Delta T\right)^{2}}{4 R_{T E G}}
$$

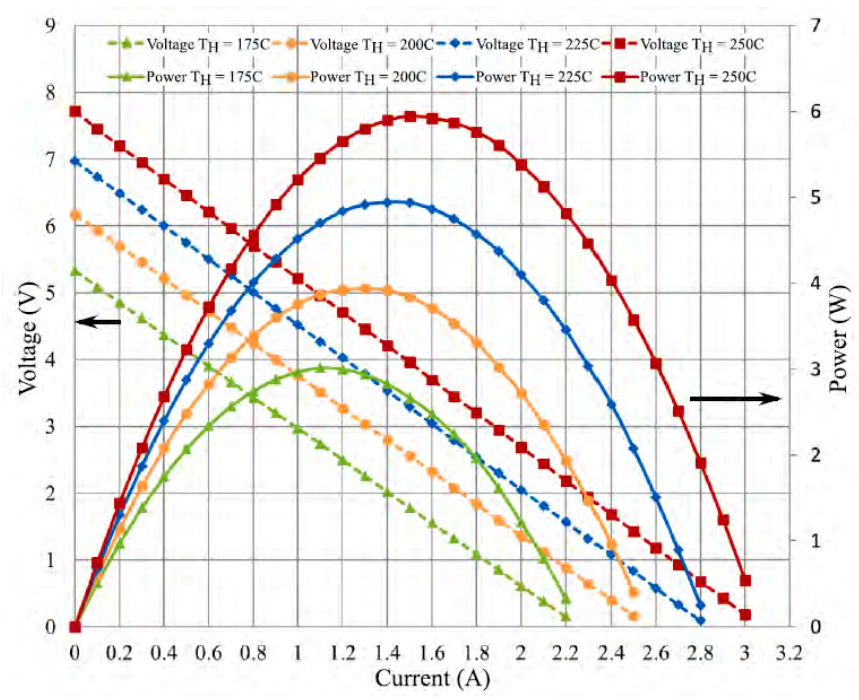

Fig. 3. Characteristic diagram of the TEG device. P-V and i-V curves obtained from the variation of temperature $(T)$ and load resistance $\left(R_{L}\right)$; results for different $T_{H}$ values with $\mathrm{T}_{\mathrm{C}}=35^{\circ} \mathrm{C}$.

Then the output power-voltage relationship $\left(P_{T E G ;}, v_{T E G}\right)$ is a parabolic function with respect to load. Typical characteristics of a TEG device for different temperatures are shown in Fig. 3. In this diagram, a TEG device has been characterized by different $T_{H}$ values while the $T_{C}$ temperature is kept at $35^{\circ} \mathrm{C}$. As seen in the diagram, there is a maximum power point (MPP) on the curve. Furthermore, since the current and voltage of the TEG vary linearly depending on the load $\left(R_{L}\right)$, the MPP can be tracked at half the value of the current $\left(i_{M P P}\right)$ and the 
voltage $\left(v_{M P P}\right)$ as shown in Fig. 3. These maximum power conditions are well known and MPPT algorithms can be used.

$$
v_{M P P}=v_{T E G}^{\max }=\frac{v_{O C}}{2} ; \quad i_{M P P}=i_{T E G}^{\max }=\frac{i_{S C}}{2}
$$

If the TEG generator is connected directly to a load resistor, the device will operate at a point on the P-V curve associated with $R_{L}$. This causes the TEG device to operate at a level defined by the load, not at the optimum operating point (MPP). However, if the $R_{L}$ load is connected to the TEG device through a Boost converter, it is possible to operate at the optimum power point by applying an MPPT algorithm on the converter control.

\section{Differential Power Processing (DPP)}

In general, series connection of multiple TEG modules is a frequently used option to increase the value of the output voltage. However, such systems generally operate under conditions of non-uniform temperature distribution, causing mismatched power losses in TEG systems. Therefore, its performance depends on the operating conditions of the TEG devices that make up the system. Different methods have been proposed in the literature (centralized systems, string-type systems and modular systems), generally supported by an MPPT converter that seeks to optimize the structure globally. But they require that the temperature differences be small so that the mismatched power losses are also low. This centralized TEG system generally incorporates a boost converter to provide power to different loads. In this way, the output voltage $v_{L}$ is adjusted by controlling the duty cycle $D$ and thus the value of MPP is maintained.

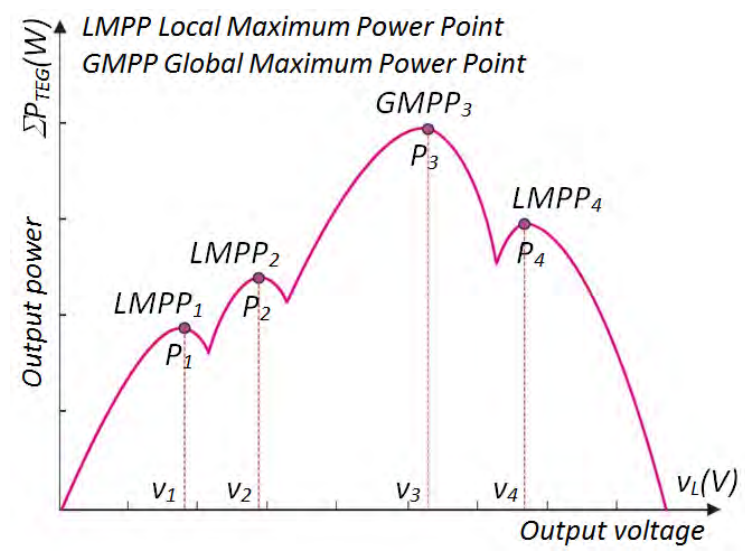

Fig. 4. Relationship between total power and output voltage provided by a TEG string-type structure under non-uniform temperature conditions.

Furthermore, in the case that the temperature differences are not low, several local maximum power points (LMPP) appear on the system's P-V curve. A detail of this behavior can be observed in Fig. 4. This phenomenon is caused by the imbalances that exist in the devices, so that the output voltage in each TEG generator will be different. Mismatch among TEG devices not only causes power losses but also creates hotspots, which will eventually affect the reliability and lifespan of the TEG modules.
If a centralized system with several TEGs connected in series is considered and it is also assumed that an ideal MPPT algorithm is developed on TEG module-array, then maximum power $P_{T}$ that can be delivered from this configuration will be:

$$
P_{T}^{\max }=\frac{\left(v_{O C 1}+v_{O C 2}+\ldots+v_{O C n}\right)^{2}}{4\left(R_{T E G 1}+R_{T E G 2}+\ldots+R_{T E G n}\right)}=\frac{\left(\sum_{i=1}^{n} v_{O C, i}\right)^{2}}{4 \sum_{i=1}^{n} R_{T E G, i}}
$$

On the other hand, if the maximum available power $P_{T E G}$ is defined in each module separately and not as all connected in series then,

$$
P_{T E G}^{\max }=\frac{v_{O C 1}{ }^{2}}{4 R_{T E G 1}}+\frac{v_{O C 2}{ }^{2}}{4 R_{T E G 2}}+\ldots+\frac{v_{O C n}{ }^{2}}{4 R_{T E G n}}=\sum_{i=1}^{n} \frac{v_{O C, i}{ }^{2}}{4 R_{T E G, i}}
$$

Furthermore, it is easy to show that $\mathrm{P}_{\mathrm{T}}{ }^{\max } \leq \mathrm{P}_{\mathrm{TEG}}{ }^{\max }$. The equality holds only if the short-circuit currents $\left(i_{S C}\right)$ are equal. This occurs since in the serial topology all TEG devices have the same $i_{T E G}$ current. From these simple preliminary observations, it can be deduced that the MPPT technique applied to each TEG module recovers more power, although its cost is higher.

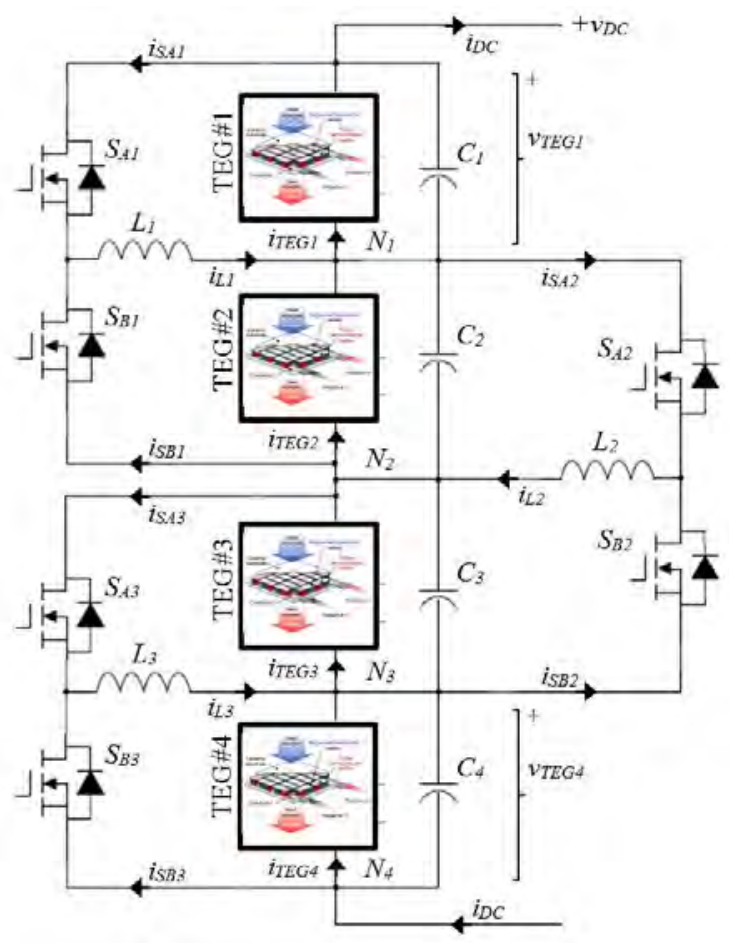

Fig. 5. Diagram of the implemented DPP architecture (bidirectional buckboost converter with synchronous switching).

The main objective of this paper has been to analyze the application of the differential-power processing architecture on a thermoelectric system composed of multiple TEG devices. The centralized-power converter processes $100 \%$ of the power generated by each TEG module, while the differential-power converter only processes a partial-power as a result of active balancing, see Fig. 5. The main advantage of this architecture 
is that it allows us to implement a control strategy that facilitates the MPPT in each TEG module. In this way, the modules can be controlled to obtain maximum power at all times. Figure 5 shows the differential power processing architecture applied to a centralized TEG structure. In the case presented here, a non-isolated architecture has been used among the different sub-modules that make up the TEG system [18]. Differential converters are connected to the TEG cells to balance the current flows between the different sub-modules. Every two cells a voltage balance circuit is connected in parallel, see Fig. 5. In other words, if there are $n$ TEG devices, there will be $n-1$ voltage balance circuits.

The principle of operation in the DPP architecture is based on the active balancing of TEG cells, through the injection or extraction of current from the connection nodes. Each DPP converter is bidirectional and maintains MPP operation on a TEG generator. This MPPT is maintained for each TEG due to the differential current provided by the DPP. Therefore, the DPP converter acts as a controllable current source and only processes a fraction of the power generated by TEG devices. In this way, conversion losses are minimized and performance is improved, compared to a global MPPT converter.

The topology proposed here consists of a bidirectional buck-boost converter with synchronous switching to balance the different TEG modules. The control strategy allows us to apply the MPPT algorithm to each TEG device independently. In steady-state, the TEG module voltage is constant and, therefore, the average current through all capacitors is zero. Applying Kirchhoff's law to node N2, eq. (10) is obtained. In turn, the currents in the $S_{B 1}$ and $S_{A 3}$ devices can be rewritten as a function of the current in each coil $L_{j}$, respectively (Fig. 5).

$$
\begin{gathered}
i_{L 2}+i_{T E G 3}=i_{T E G 2}+i_{S B 1}+i_{S A 3} \\
i_{S B 1}=i_{L 1}\left(1-D_{1}\right) \\
i_{S A 3}=i_{L 3} D_{3}
\end{gathered}
$$

Thus, for any connection node $N_{j}$, the expression can be generalized as:

$$
i_{L j}+i_{T E G, j+1}=i_{T E G j}+i_{L, j-1}\left(1-D_{j-1}\right)+i_{L, j+1} D_{j+1}
$$

In the first node, the equation will be adjusted ignoring the term $i_{L, j-1}$ while in the equation of the last node the term $i_{L, j+I}$ will be omitted. In this equation, it can be seen that the average current of the inductor $i_{L j}$ in a TEG device depends in turn on the average currents in the other inductors $i_{L, j+1}$ and $i_{L, j-1}$. Finally, each converter controls the differential current through its duty cycle $D_{j}$. Also, the duty cycle value is a function of the voltages across the TEG modules (14),

$$
D_{j}=\frac{v_{T E G, j}}{v_{T E G, j}+v_{T E G, j+1}}
$$

where $D_{j}$ is the duty cycle in the $j t h$ DPP converter, while $v_{T E G, j}$ and $v_{T E G, j+1}$ are the voltages of the $j$ th submodule and $j+1, t h$ submodule, respectively. In this way, in ideal scenarios or with low unbalances, the duty cycle of the converters will be close to the value $D_{j}=0,5$. The objective of the control algorithm is to find the value of the duty cycle $\left(D_{1}, D_{2} \ldots, D_{j}, D_{j+1}\right)$ that maximizes the output power in the system. The control loop is based on a $\mathrm{P} \& \mathrm{O}$ algorithm. The purpose of the control is to find the only combination of current values $\left(i_{T E G, j}\right)$ and duty cycle ratios $\left(D_{j}\right)$ that maximize the overall output power. Thus, the control algorithm performs several iterations until all the TEG modules find the proper duty cycle that maximizes power. An advanced mathematical analysis of the presented DPP topology, as well as a more detailed development of the structure in matrix format, can be seen in [18].

\section{Simulation Results}

To analyze the advantages and drawback of differentialpower architecture in a series-connected TEG topology, the circuit shown in Fig. 5 has been modelled and simulated. The DPP converter consists of a non-isolated bidirectional buckboost converter, which operates as a current source. The control technique has been evaluated by extensive simulation, using Matlab-Simulink software. It must be considered that the dynamics of TEG devices is relatively low. On the other hand, the duty cycle control has been implemented using a slidingmode current control. This control technique has a small-time response. The purpose of the system is to provide maximum power (voltage and current) to the DC bus, even under nonuniform temperature conditions in the TEG-array.

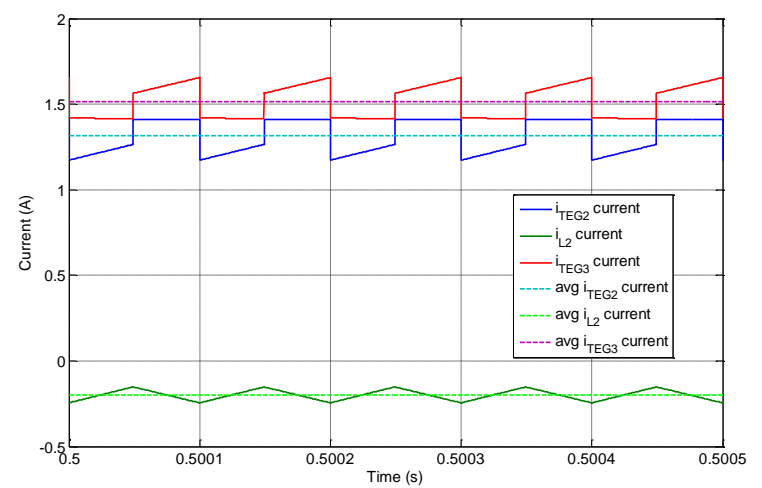

Fig. 6. Currents at node $\mathrm{N} 2$ (parameters $i_{T E G 2}, i_{T E G 3}, i_{L 2}$ ); instantaneous and average values. Assumption considered $\mathrm{i}_{\mathrm{L} 2}<0$ according to the current criteria adopted.

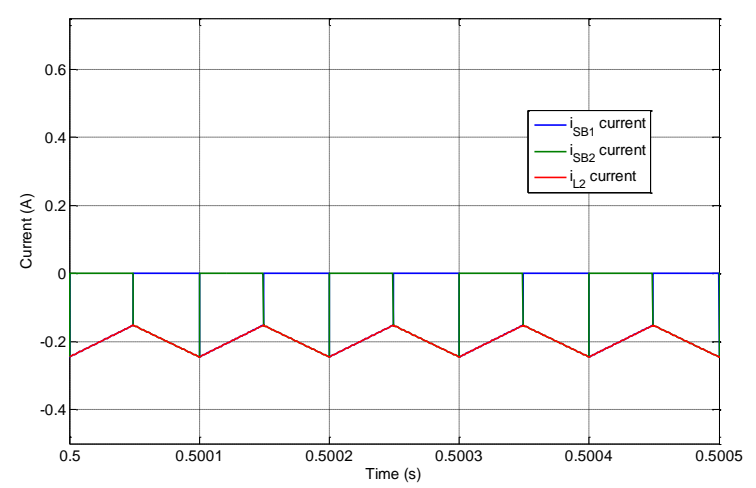

Fig. 7. Currents in the DPP converter associated with node 2 (parameters $i_{S B 1}, i_{L 2}, i_{S B 2}$ ). Assumption considered $i_{\mathrm{L} 2}<0$ according to the current criteria adopted.

Differential-power converters have a switching frequency of $\mathrm{f}_{\mathrm{SW}}=10 \mathrm{kHz}$ and a coil of $\mathrm{L}_{\mathrm{j}}=1,5 \mathrm{mH}$. In this model, 
resistance in series with the inductor $\left(\mathrm{R}_{\mathrm{Lj}}=0,1 \Omega\right)$ has been included to represent the losses. In Fig. 6 and 7 it is possible to observe the different currents associated to node $\mathrm{N} 2$ and to the DPP converter, respectively. All these variables are related to node $\mathrm{N} 2$. In this case, it is observed that $\mathrm{i}_{\mathrm{L} 2}<0$, that is, the subconverter removes current at node $\mathrm{N} 2$, see Fig. 6. As a consequence of active balancing, the operating point in the TEG2 device $\left(v_{T E G 2} ; i_{T E G 2}\right)$ is different from the operating point in the TEG3 device $\left(v_{T E G 3} ; i_{T E G 3}\right)$. Likewise, it has been considered that the TEG devices have a temperature, on the hot side, of $\mathrm{T}_{\mathrm{H} 2}=200^{\circ} \mathrm{C}$ and $\mathrm{T}_{\mathrm{H} 3}=250^{\circ} \mathrm{C}$, respectively. In both cases, the value of $\mathrm{T}_{\mathrm{C}}=35^{\circ} \mathrm{C}$, see diagram in Fig. 3. In the case studied, the following values have been obtained: $\mathrm{i}_{\mathrm{TEG} 2 \text { (avg) }}=+1,317 \mathrm{~A} ; \quad \mathrm{i}_{\mathrm{TEG} 3 \text { (avg) }}=+1,515 \mathrm{~A} ; \quad \mathrm{i}_{\mathrm{L} 2 \text { (avg) }}=-0,1986 \mathrm{~A} ;$ $i_{\mathrm{SA2} \text { (avg) }}=-0,0993 \mathrm{~A} ; i_{\mathrm{SB} 2 \text { (avg) }}=-0,0993 \mathrm{~A}$. Also, the voltages on the TEG devices are: $\mathrm{v}_{\mathrm{TEG} 2 \text { (avg) }}=+3,02 \mathrm{~V} ; \mathrm{v}_{\mathrm{TEG} 3 \text { (avg) }}=+3,92 \mathrm{~V}$. In this way, both TEG cells are operating in their own MPP. This phenomenon is more representative when the number $N$ of thermocouple junctions within the TEG device increases.

\section{CONCLUSIONS}

This paper has presented a differential power processing (DPP) topology applied to series-connected thermoelectric generators. The DPP converter is based on the active balancing principle. The main objective has been to allow each submodule to reach its maximum power point (MPP) more quickly under non-uniform temperature conditions. In this way, the overall efficiency of the TEG system is improved. The advantage of the DPP converter over the Full-converter is that now the nominal power of the converter can be a small fraction of the TEG sub-module power, instead of the total power of the system. In this way, as a partial solution, the DPP subconverter allows us to combine the active balance with the local energy optimization in each small thermoelectric generator, being a more efficient alternative than the global optimizer. Matlab-Simulink has been the software used to develop the TEG module-array and analyze its performance. The simulation results obtained have been satisfactory.

\section{ACKNOWLEDGMENT}

The authors would like to thank the support of Government of Aragon and the European Union project T28_17R, "building Aragon from Europe". This work was supported in part by the Spanish MINECO under Grant RTC-2015-3358-5. The authors want to thank the support of the IDI project IDI-20190433 IHIESS \& SAHI-RIS3-, project LMP_16_18 from the Aragon government RIS3 program, and the European Union, as well as the support of Sociedad Ibérica de Construcciones Eléctricas.

\section{REFERENCES}

[1] N. Jaziri, A. Boughamoura, J. Müller et al., "A comprehensive review of Thermoelectric Generators: Technologies and common applications". Energy Reports. December 2019, in press.

[2] S. Twaha, J. Zhu, Y. Yan and B. Li, "A comprehensive review of thermoelectric technology: Materials, applications, modelling and performance improvement". Renewable and Sustainable Energy Reviews. vol. 65, November 2016; pp. 698-726.
[3] R. Saidur, M. Rezaei, W.K. Muzammil, M.H. Hassan, S. Paria and M. Hasanuzzaman, "Technologies to recover exhaust heat from internal combustion engines". Renewable and Sustainable Energy Reviews. Elsevier Science Direct. vol. 16, October 2012, pp. 5649-5659.

[4] L. Heber \& J. Schwab, "Modelling of a thermoelectric generator for heavy-duty natural gas vehicles: Techno-economic approach and experimental investigation". Applied Thermal Engineering. Elsevier Science Direct. vol. 174, June 2020; pp. 1-12.

[5] S.R. Yan, H. Moria, S. Asaadi, H.S. Dizaji, S. Khalilarya and K. Jermsittiparsert, "Performance and profit analysis of thermoelectric power generators mounted on channels with different cross-sectional shapes". Applied Thermal Engineering. Elsevier Science Direct. vol. 176, July 2020; pp. 1-12.

[6] M. Gao, C. Su, J. Cong, F. Yang, Y. Wang and P. Wang, "Harvesting thermoelectric energy from railway track". Energy, Elsevier Science Direct. vol. 180. August 2019, pp. 315-329.

[7] L. Kütt, J. Millar, A. Karttunen, M. Lehtonen and M. Karppinen, "Thermoelectric applications for energy harvesting in domestic applications and micro-production units. Part I: Thermoelectric concepts, domestic boilers and biomass stoves". Renewable and Sustainable Energy Reviews. vol. 98, December. 2018, pp. 519-544.

[8] A. Dewan, S.U. Ay, M. Nazmul and H. Beyenal, "Alternative power sources for remote sensors: A review". Journal of Power Sources. Elsevier Science Direct. vol. 245, January 2014, pp. 129-143.

[9] Q. Wan, Y.K. The, Y. Gao and P.K.T. Mok, "Analysis and Design of a Thermoelectric Energy Harvesting System With Reconfigurable Array of Thermoelectric Generators for IoT Applications". IEEE Transactions on Circuits and Systems I: Regular Papers. IEEExplore Digital Library. vol. 64, No 9. September 2017, pp. 2346-2358.

[10] M. Hasani \& N. Rahbar, "Application of thermoelectric cooler as a power generator in waste heat recovery from a PEM fuel cell - An experimental study". International Journal of Hydrogen Energy, Elsevier Science Direct. vol. 40, issue 43. November 2015, pp. 15040-15051.

[11] D. Champier, "Thermoelectric generators: A review of applications". Energy Conversion and Management. Elsevier Science Direct. vol. 140, May 2017; pp. 167-181.

[12] M. Bond and J.D. Park, "Current-Sensorless Power Estimation and MPPT Implementation for Thermoelectric Generators". IEEE Transactions on Industrial Electronics, IEEExplore Digital Library. Vol. 62, issue 9. September 2015, pp. 5539-5548.

[13] R.Y. Kim, J.S. Lai, B. York and A. Koran, "Analysis and Design of Maximum Power Point Tracking Scheme for Thermoelectric Battery Energy Storage System". IEEE Transactions on Industrial Electronics, IEEExplore Digital Library. vol. 56, issue 9. Sept. 2019, pp. 3709-3716.

[14] A. Montecucco \& A.R. Knox, "Maximum Power Point Tracking Converter Based on the Open-Circuit Voltage Method for Thermoelectric Generators". IEEE Transactions on Power Electronics, IEEExplore Digital Library. vol. 30, No 2. February 2015, pp. 828-839.

[15] J.D. Park, H. Lee and M. Bond, "Uninterrupted thermoelectric energy harvesting using temperature-sensor-based maximum power point tracking system". Energy Conversion and Management. Elsevier Science Direct. vol. 98, October 2014; pp. 233-240.

[16] B. Yang, J. Wang, X. Zhang, M. Zhang, H. Shu, S. Li, T. He; L. Yang and $\mathrm{T}$. Yu, "MPPT design of centralized thermoelectric generation system using adaptive compass search under non-uniform temperature distribution condition". Energy Conversion and Management. Elsevier Science Direct. vol. 199, November 2019; pp. 1-14.

[17] S. Qin, S.T. Cady, A.D. Domínguez-García \& R.C.N. Pilawa-Podgurski, "A distributed approach to MPPT for PV sub-module differential power processing". IEEE Energy Conversion Congress and Exposition. IEEExplore Digital Library. September 2013, Denver, CO (USA); pp. 2778-2785.

[18] J.S. Artal-Sevil, J.A. Domínguez, C. Bernal-Ruiz and A. Coronado, "Power Flow Control through Differential Power Processing to improve reliability in hybrid systems based on PEM-Fuel Cell". $15^{\text {th }}$ International Conference on Ecological Vehicles and Renowable Energies (EVER). IEEExplore Digital Library. September 2020; Monaco; in-press. 